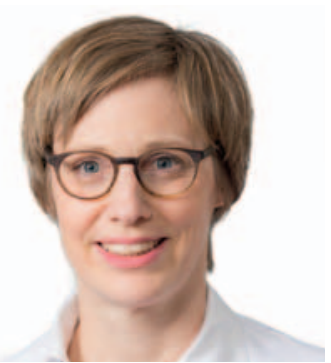

Ameli Gabel-Pfisterer

Klinikum Ernst von Bergmann gGmbH,

Potsdam, Deutschland

\title{
Augenverletzungen durch private Feuerwerks- und Knallkörper
}

\author{
Ausgewählt und kommentiert von \\ Dr. Ameli Gabel-Pfisterer (Potsdam)
}

In jeder Silvesternacht verletzen sich in Deutschland tausende Menschen durch privat gezündete Feuerwerks- und Knallkörper. Genaue Zahlen für Deutschland sind noch nicht bekannt. Internationale Studien legen nahe, dass die Hände, der Kopf und die Augen besonders häufig betroffen sind [1]. Auch wenn oberflächliche Lid-, Bindehaut-, oder Hornhautverletzungen häufig ohne bleibende Schäden heilen, können sich tief einbrennende oder mit hoher Energie auftreffende Feuerwerks- und Knallkörper zu Verletzungen mit einer schlechten Visusprognose führen. Bulbusrupturen oder penetrierende Verletzungen durch explodierende Sprengkörper können trotz intensiver Erstversorgung den Verlust eines Auges bedeuten. Wie häufig sind solche schweren Verletzungen? Wer sind die Unfallopfer? Wie liegt die Bundesrepublik Deutschland im internationalen Vergleich? Und wie können Augenverletzungen durch Feuerwerks- und Knallkörper vermieden werden?

Die ausgewählten Artikel beleuchten diese Punkte genauer:

Die Arbeitsgruppe aus der Universitätsaugenklinik Leipzig berichtet aus den Jahren 2005 bis 2013 von 122 Patienten, die wegen Feuerwerks- und Knallkörperverletzungen behandelt wurden. Mehr als ein Drittel der Patienten hatte eine schwere Bulbusverletzung, wobei trotz oft mehrfacher Operation jedes fünfte Auge erblindete [2]. Die Metaanalyse von Wisse, die Daten aus 26 internationalen Studien zusammenführt, zeigt, dass rund jeder fünfte Patient eine schwere Augenverletzung erlitt, die nahezu in allen Fällen zu einem Visusverlust auf unter 0,2 führte [3]. Die Arbeitsgruppe um Chang berichtet aus einem US-amerikanischen Trauma-Zentrum bei operativ versorgten Bulbusrupturen nach Feuerwerks- oder Knallkörperverletzung in 17\% der Fälle ebenfalls von sehr schlechten Visusprognosen [4].

\section{Alters- und und Geschlechterverteilung}

Alle Artikel zeigen deutlich, dass männliche Kinder, Jugendliche und junge Erwachsene bis 25 Jahre, die am Beginn ihres Berufslebens stehen, bei den Verletzten mit rund 30\% überrepräsentiert sind [2-5]. Wenn man bedenkt, dass bei rund 15\% der Patienten beide Augen, und in einigen Fällen auch das Gesicht und die Hände, betroffen sind, wie die Ergebnisse unserer deutschlandweiten Umfrage zu Silvester 2016/2017 zeigen [5], kann man sich persönliche Dramen, aber auch erhebliche solidarisch getragene Folgekosten vorstellen.

\section{KARGER}

Fax +497614520714

information@karger.com

www.karger.com (c) 2018 S. Karger GmbH, Freiburg
Dr. Ameli Gabel-Pfisterer

Klinikum Ernst von Bergmann gGmbH

Charlottenstraße 72

14467 Potsdam, Deutschland

agabel-pfisterer@klinikumevb.de 


\section{Zuschauer und Passanten}

Dramatischerweise haben viele Unfallopfer den Feuerwerks- oder Knallkörper nicht selbst gezündet. Die Zahlen variieren je nach Studie, wahrscheinlich in Abhängigkeit der Fragestellung, zwischen $30 \%$ und $80 \%$, zeigen in jedem Fall aber ein sehr hohes Maß an Fremdgefährdung [2-5]. Die Studie von Chang beschreibt gerade bei diesen Patienten ein besonders hohes Risiko für schwerste Bulbusrupturen [4].

\section{Prävention}

Ob das Verkaufsverbot für Feuerwerkskörper der Kategorie 2 an Personen unter 18 Jahren einen ausreichenden Schutz Minderjähriger darstellt, darf angesichts dieser Zahlen angezweifelt werden. Zudem läuft die Gesetzeslage derzeit einer aus augenärztlicher Sicht sinnvollen Regelung leider zuwider. So zeigt die Arbeit der Leipziger Arbeitsgruppe einen Anstieg von Augenverletzungen infolge der Anpassung des Sprengstoffgesetzes an die im Umgang mit Schwarzpulver großzügigere Europäische Richtlinie im Jahr 2009 [2].

In einigen Ländern wurden unterschiedlich bewertete Versuche unternommen, um die Zahlen von feuerwerksbedingten Augenverletzungen durch Aufklärung $[6,7]$ oder durch Mitverkauf von
Schutzbrillen zu reduzieren [8]. Einen effektiven Schutz scheint nur eine Beschränkung privat gezündeter Feuerwerke zu bieten: In Ländern, die sich auf offizielle Feuerwerke beschränken, kommen Feuerwerks-und Knallkörper-assoziierte Augenverletzungen 87mal seltener vor [3].

\section{Fazit}

Privat gezündete Feuerwerke sind gefährlich, auch wenn sie nicht selbst gezündet werden!

Unsere Arbeitsgruppe aus Freiburg und Potsdam, mit allen beteiligten Augenärzten und Augenärztinnen, die uns bei der deutschlandweiten Umfrage unterstützen, möchte eine stabile Datenbasis für eine breite Diskussion um ein Verbot privat genutzter Feuerwerks- und Knallkörper schaffen. Wir unterstützen damit den Aufruf des International Council of Ophthalmology (ICO) aus dem Jahr 2016, das aus gutem Grund ein weltweites Verbot privat genutzter Feuerwerke fordert [9].

\section{Disclosure Statement}

Hiermit erkläre ich, dass keine Interessenskonflikte in Bezug auf diesen Beitrag bestehen.

\section{Literatur}

1 United States Consumer Product Safety Commision: National Electronic Injury Surveillance System (NEISS): 2015 NEISS Data Highlights. www.cpsc.gov/s3fs-public/2015\%20Neiss\% 20data\%20highlights.pdf (Zugriff 21.12.2017).

2 Unterlauft JD, Wiedemann P, Meier P: Bulbustraumata durch Feuerwerkskörper von 2005 bis 2013. Klin Monatsbl Augenheilkd 2014; 231:915-920.

3 Wisse RPL, Bijisma WR, Stilma JS: Ocular firework trauma: a systematic review on incidence, severity, outcome and prevention. Br J Ophthalmol 2010;94:1586-1591.
4 Chang IT, Prendes MA, Tarbet KJ, et al.: Ocular injuries from fireworks: the 11-year experience of a US level I trauma center. Eye (Lond) 2016;30:1324-1330.

5 Gabel-Pfisterer A, Böhringer D, Agostini H, et al.: Augenverletzungen durch Feuerwerksoder Knallkörper an Silverster 2016/2017: Eine Umfrage unter deutschen Augenkliniken. Jahrestagung der Deutschen Ophthalmologischen Gesellschaft 2017: PDo08-04.

6 D'Argenio P, Cafaro L, Santonastasi F, et al.: Capodanno Senza Danno: the effects of an intervention program an fireworks injuries in Naples. Am J Public Health 1996;86:84-86.
7 Thygesen J: Ocular injuries caused by fireworks. 25 years of experience with preventive campaigns in Denmark. Acta Ophthalmol Scand 2000;78:1-2.

8 Bull N: Legislation as a tool to prevent firework-related eye injuries. Acta Ophthalmol 2011;89:e654-e655.

9 International Council of Ophthalmology: ICO endorses global ban of fireworks. www.icoph.org/ downloads/ICOPositionPaper-FireworksBanMay2016.pdf(Zugriff 18.12.2017). 